\title{
Somatic mutations in telomerase promoter counterbalance germline loss-of-function mutations
}

\author{
Lindley Maryoung, ${ }^{1}$ Yangbo Yue, ${ }^{2}$ Ashley Young, ${ }^{1}$ Chad A. Newton, ${ }^{1,3}$ Cindy Barba, ${ }^{2}$ Nicolai S.C. van Oers, ${ }^{4}$ \\ Richard C. Wang, ${ }^{2}$ and Christine Kim Garcia',3 \\ 'McDermott Center for Human Growth and Development, ${ }^{2}$ Department of Dermatology, ${ }^{3}$ Department of Internal Medicine, Pulmonary and Critical Care Medicine, and ${ }^{4}$ Department of Immunology, \\ UT Southwestern Medical Center, Dallas, Texas, USA.
}

\begin{abstract}
Germline coding mutations in different telomere-related genes have been linked to autosomal-dominant familial pulmonary fibrosis. Individuals with these inherited mutations demonstrate incomplete penetrance of clinical phenotypes affecting the lung, blood, liver, skin, and other organs. Here, we describe the somatic acquisition of promoter mutations in telomerase reverse transcriptase (TERT) in blood leukocytes of approximately $5 \%$ of individuals with inherited loss-of-function coding mutations in TERT or poly(A)-specific ribonuclease (PARN), another gene linked to telomerase function. While these promoter mutations were initially identified as oncogenic drivers of cancer, individuals expressing the mutations have no history of cancer. Neither promoter mutation was found in population-based cohorts of similar or advanced age. The TERT promoter mutations were found more frequently in cis with the WT allele than the TERT coding sequence mutation. EBVtransformed lymphoblastoid B cell lines (LCLs) derived from subjects with TERT promoter mutations showed increased telomerase expression and activity compared with cell lines from family members with identical coding mutations. TERT promoter mutations resulted in an increased proliferation of LCLs and demonstrated positive selection over time. The persistence and recurrence of noncoding gain-of-function mutations in these cases suggests that telomerase activation is not only safely tolerated but also advantageous for clonal expansion.
\end{abstract}

\section{Introduction}

Telomerase is a reverse transcriptase that extends the length of repetitive DNA sequences at the ends of chromosomes (1). In contrast to normal tissue, in which telomerase activity is absent, most human tumors (85\%-90\%) constitutively express telomerase (2). Activating germline mutations in the telomerase reverse transcriptase (TERT) promoter are some of the most common mutations found in human cancer, occurring frequently in bladder cancer, melanoma, and glioblastoma (3). The most common TERT promoter mutations are located at positions -124 and -146 upstream of the TERT coding start site. These mutations create a transcription binding site for GABP, an ETS transcription factor (4), and lead to increased TERT expression, telomerase activity, telomere length, and tumor formation $(5,6)$.

Coding mutations in TERT as well as other genes in the telomere pathway (telomerase RNA component [TERC], poly (A)specific ribonuclease $[P A R N]$, regulator of telomere elongation helicase 1 [RTEL1], and nuclear assembly factor 1 ribonucleoprotein [NAF1]) have been found in patients with familial pulmonary fibrosis and idiopathic pulmonary fibrosis (IPF) (7-10). Pathogenic rare variants in TERT are found in approximately $15 \%$ of familial cohorts and approximately $3 \%$ of sporadic IPF patients; mutations are more commonly found in TERT than in any other gene. Additionally, all pathogenic rare variants lead to reduced protein function, decreased telomerase enzymatic activity, and shortened telomere lengths.

Conflict of interest: The authors have declared that no conflict of interest exists. Submitted: October 10, 2016; Accepted: December 20, 2016.

Reference information: / Clin Invest. 2017;127(3):982-986.

https://doi.org/10.1172/JCI91161
While adult-onset pulmonary fibrosis is common, the clinical spectrum of extra-pulmonary manifestations is broad and overlaps dyskeratosis congenita (DC). We hypothesized that the acquisition of somatic gain-of-function promoter mutations might counteract the effect of germline loss-of-function mutations.

\section{Results and Discussion}

The TERT promoter was sequenced from DNA isolated from blood leukocytes of 199 subjects, each with a previously identified heterozygous germline mutation in 1 of 4 telomere-related genes (TERT, TERC, PARN, and RTEL1, Supplemental Table 1; supplemental material available online with this article; doi:10.1172/ JCI91161DS1) associated with pulmonary fibrosis. The individuals, $50 \%$ of whom were male, ranged in age from 15 to 84 years (mean $51 \pm 16$ years). Table 1 lists the 10 subjects (5\%) in whom a TERT promoter mutation was found. All but 1 of these 10 subjects was non-Hispanic white (NHW), all but 1 was male, and the mean age was $54 \pm 7$ years. Nine of one hundred thirty-seven $(6.5 \%)$ with a germline mutation in TERT and one of thirty-two (3.1\%) with a germline mutation in PARN had one of two different TERT promoter mutations $(-124 \mathrm{C}>\mathrm{T}$ and $-146 \mathrm{C}>\mathrm{T})$ in blood leukocytes. There was no history of cancer in these 10 individuals. Most had pulmonary fibrosis, one was asymptomatic, and one was lost to follow up. We found no difference in leukocyte telomere lengths in subjects with TERT promoter and coding mutations compared with family members with an identical coding mutation as measured by quantitative PCR (qPCR) or flow-FISH (data not shown). The total white blood cell counts of the TERT promoter mutation carriers were within the normal range, although some of the lym- 
A
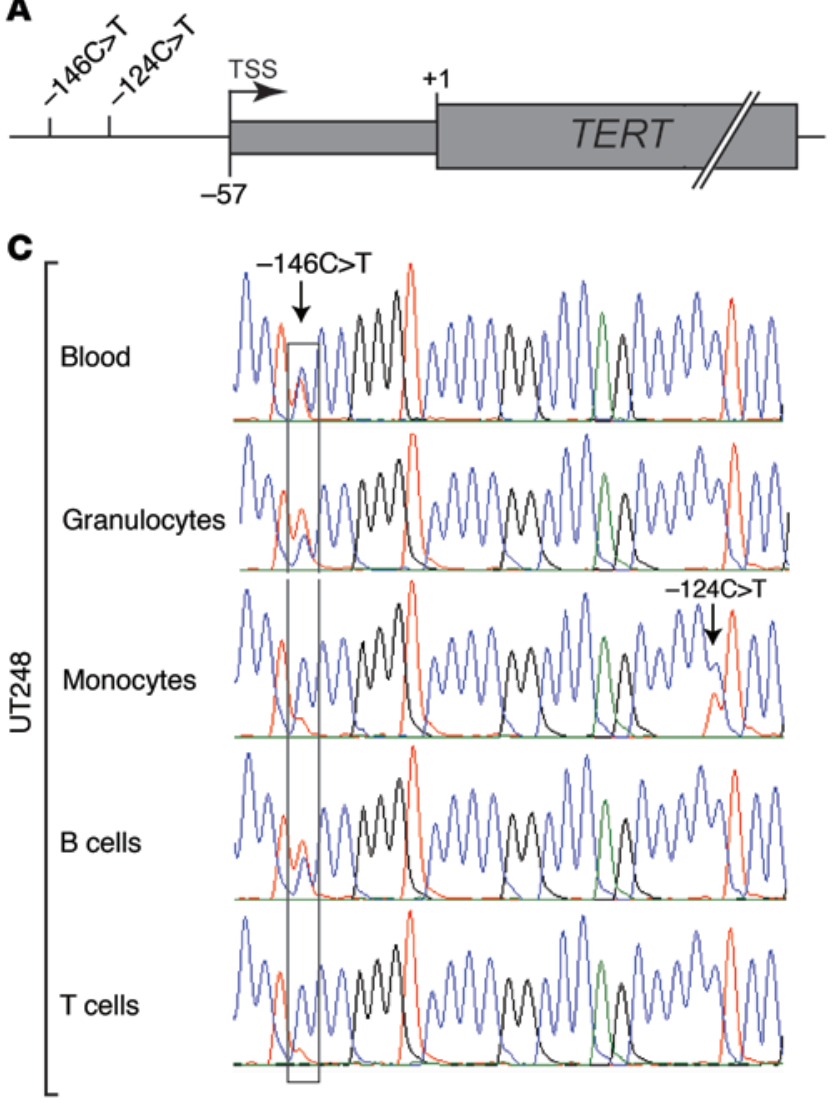

B

Control

UT248

UT2210

UT387

UT2923

UT2482
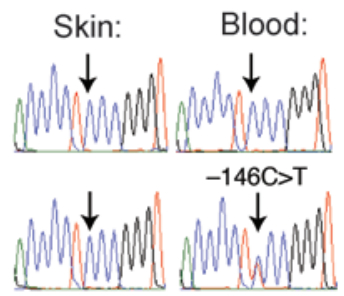

$-1460>$

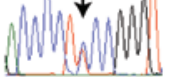

$-146 \mathrm{C}>\mathrm{T}$.

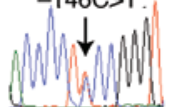

$-124 \mathrm{C}>\mathrm{T}$
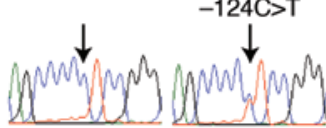

$-124 \mathrm{C}>\mathrm{T}$
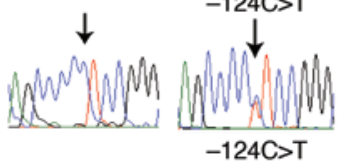

UT248

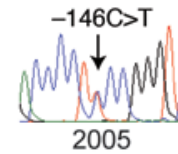

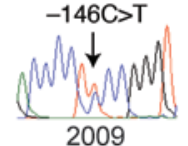

Figure 1. Somatic gain-of-function TERT promoter mutations are found in circulating leukocytes of patients with heterozygous germline TERT coding mutations. (A) Schematic of the TERT gene with the 2 gain-of-function promoter mutations located at -124 and -146 relative to the TERT CDNA start site. TSS, transcription start site. (B) Sanger sequence tracings of the TERT promoter amplified from skin and blood from unrelated subjects. (C) Flow sorting of blood leukocytes from 1 subject (UT248) shows different peak heights of the 2 different promoter mutations in various cell types. The $-146 C>T$ promoter mutation is boxed; the $-124 C>T$ promoter mutation is marked by an arrow. (D) Sanger sequence tracings of the TERT promoter $-146 C>T$ mutation amplified from independent blood samples collected from 1 subject over time. phocyte subsets were variably low, consistent with the spectrum of immunological abnormalities found in these individuals (Supplemental Tables 2 and 3). Several had evidence of macrocytosis, which is commonly seen in pulmonary fibrosis patients with germline telomere-related gene mutations (11).

The promoter mutations in TERT were not found in DNA isolated from skin fibroblasts or buccal epithelium (Table 1 and Figure 1B). As would be expected with somatic mutations, none of the progeny of the individuals listed in Table 1 were found to have the promoter mutation (data not shown).
To determine the frequency of the TERT promoter mutations in blood from control subjects, we screened the population-based Dallas Heart Study cohort (Supplemental Figure 1). None of the 2,611 subjects (mean age of $50 \pm 11$ years, including 895 NHW and 1,102 males) was found to carry either of these 2 TERT promoter mutations. Thus, 9 of the 179 NHW telomererelated mutation carriers and none of the 895 NHW control subjects were found to have a TERT promoter mutation $(P=8.36$ $\times 10^{-8}$ by Fisher's exact test). In addition, none of the 132 individuals from advanced-age control populations, ranging in age

Table 1. Blood leukocyte TERT promoter somatic mutations found in subjects with various heterozygous germline mutations in telomere-related genes

\begin{tabular}{|c|c|c|c|c|c|c|c|}
\hline Subject ID & Age (yr) & Sex & $\begin{array}{l}\text { TERT promoter } \\
\text { somatic mutation }\end{array}$ & Blood & Skin & Germline coding sequence mutation & Clinical phenotype \\
\hline UT2923 & 61 & M & $-124 C>T$ & Present & Absent & TERT c.1710G>T (Lys570Asn, exon 3) & Pulmonary fibrosis, s/p lung transplant \\
\hline UT3224 & 47 & M & $-124 C>T$ & Present & Absent & TERT c.1895C>T (Pro632Leu, exon 4) & Pulmonary fibrosis \\
\hline UT387 & 52 & M & $-124[>T$ & Present & Absent & TERT c.2240delT (Val747Valfs, exon 6) & Died, pulmonary fibrosis \\
\hline UT248 & 47 & $\mathrm{~F}$ & $-146[>T /-124 C>T$ & Present & Absent & TERT c.2594G>A (Arg865His, exon 9) & Pulmonary fibrosis \\
\hline UT2482 & 50 & M & $-124 C>T$ & Present & ND & TERT c.2627T>A (Phe883lle, exon 10) & Died, pulmonary fibrosis \\
\hline UT3410 & 63 & M & $-124 C>T$ & Present & ND & TERT c.2991delG (Val997Valfs, exon 13) & Pulmonary fibrosis \\
\hline UT986 & 64 & M & $-124 C>T$ & Present & ND & PARN c.529C >T (GIn177Ter) & Died, sepsis \\
\hline
\end{tabular}

F, female; M, male; ND, not determined; s/p, status post. 
A
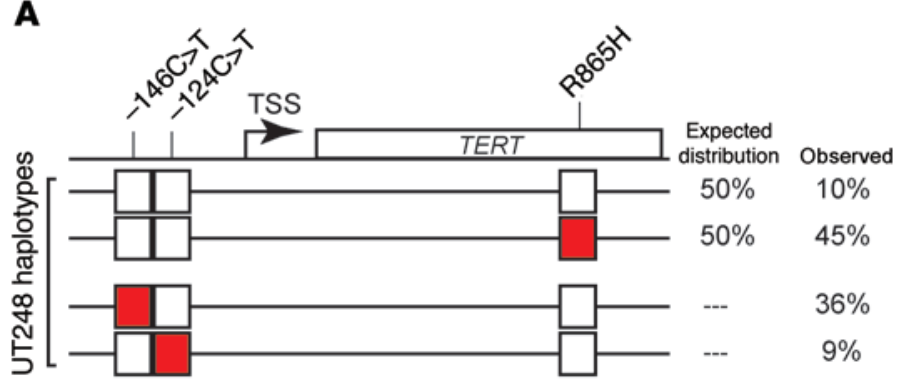

C

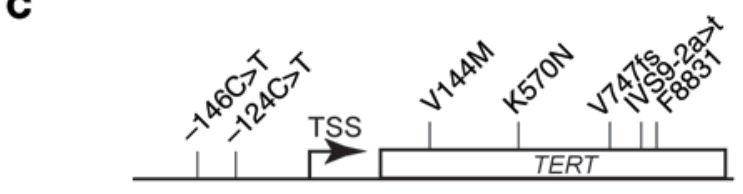

Expected
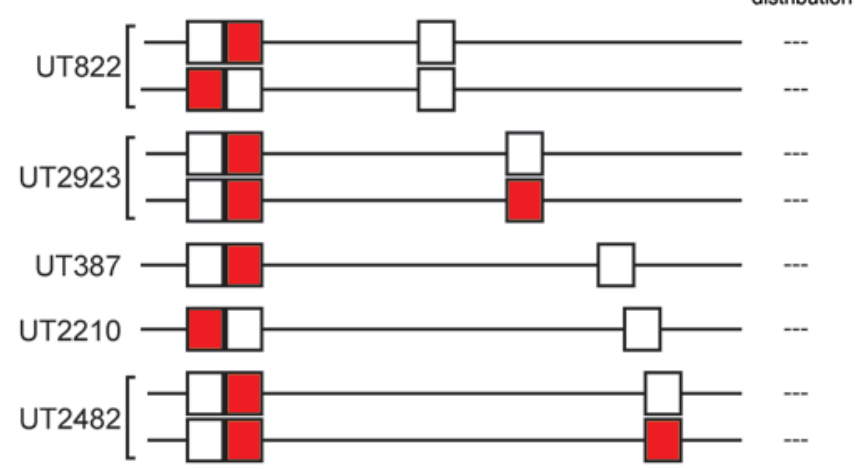

from 98 to 108 years, was found to have either of these promoter mutations (data not shown).

Blood samples from the subjects were freshly collected, and granulocytes, monocytes, B cells, and T cells were isolated by flow sorting. DNA sequencing was used to determine the relative frequency of the promoter mutation in TERT. Granulocytes and B cells from subject UT248 showed a higher peak of the mutant T nucleotide at position -146 (red) than did the WT C nucleotide (blue) (Figure 1C). A lower level of the mutant $\mathrm{T}$ nucleotide was detected in monocytes and $\mathrm{T}$ cells. Remarkably, a C-to-T substitution was noted at position -124 specifically in the monocyte fraction, which was not detected in the initial sequencing. For 2 individuals (UT2031 and UT3224), the small $-124 \mathrm{C}>\mathrm{T}$ promoter mutation peak was more readily apparent in DNA isolated from granulocyte, monocyte, or B cell fractions than in DNA from whole blood (Supplemental Figure 2). Independent blood samples were obtained from 1 individual (UT248) over a 10-year period, and the $-146 \mathrm{C}>\mathrm{T}$ peak was present in all samples (Figure 1D).

To determine whether the promoter mutations were linked in cis or trans to the heterozygous TERT coding mutations, haplotypes (spanning up to $28.8 \mathrm{~kb}$ ) were created by limiting dilution PCR. Haplotypes for individual UT248 are shown in Figure 2A. Both mutant TERT promoter sequences were amplified as single peaks from very dilute DNA samples, confirming the cell fractionation result seen in Figure 1C. In each case, the promoter mutation was found in cis with the WT TERT cDNA sequence (Figure 2B). The promoter mutation was more commonly in cis with the WT $T E R T$ CDNA sequence than the TERT coding sequence mutation across different individuals (Figure 2C, Supplemental Figure 3,

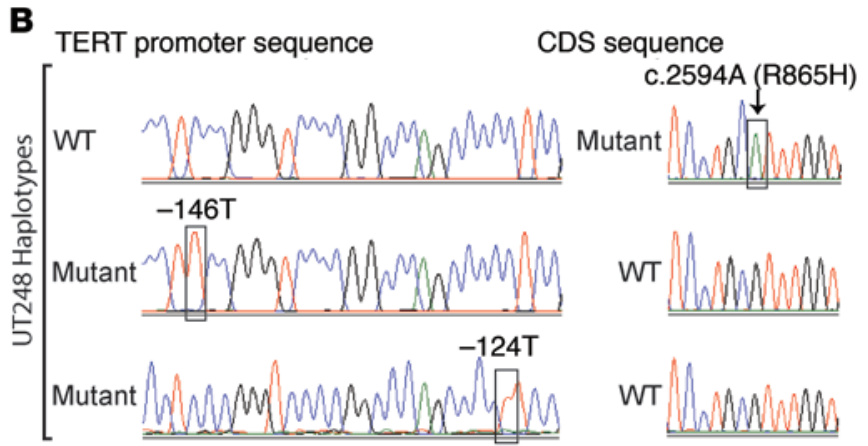

Figure 2. TERT promoter mutations are frequently found in cis with the WT TERT allele in blood leukocyte DNA haplotypes. (A) Haplotypes of the TERT gene constructed by limiting dilution PCR of genomic DNA isolated from the blood of 1 subject (UT248). WT and mutant TERT alleles are indicated by white and red boxes, respectively. The observed frequency of each haplotype is indicated. (B) Sanger sequence tracings of the TERT promoter and coding regions. Mutations are boxed. CDS, coding sequence. (C) Haplotypes constructed from limiting dilution PCR of DNA from 5 additional individuals. Only the haplotypes containing promoter mutations are shown. Percentages denote the observed frequencies of the indicated haplotype.

and Supplemental Table 4). Thus, there is a bias toward selection of cells in which the activating promoter mutation is driving the expression of the WT, but not the mutant, telomerase allele.

We used cell lines to test whether the promoter mutation found in cis with the WT allele is associated with increased telomerase activity. The EBV-transformed lymphoblastoid B cell line (LCL) derived from the individual carrying the $\mathrm{C}>\mathrm{T}$ mutation at position -146 in the TERT promoter (subject UT248) had higher telomerase activity and higher human TERT gene expression (Figure 3, A and B) than did family members with the same TERT coding sequence mutation. The mutant $-146 \mathrm{C}>\mathrm{T}$ peak was less prominent in slowly proliferating cells than in rapidly proliferating cells after approximately 1 week (Figure 3C). The relative abundance of the $-146 \mathrm{C}>\mathrm{T}$ mutation was much higher after 46 weeks in culture, indicating a preferential selection for this mutant allele (Figure 3D). LCLs isolated from carriers with only the TERT coding sequence mutation did not acquire the TERT promoter mutations over time (data not shown). We also noted increased telomerase activity and human TERT (hTERT) expression for subject UT3410, who carries the $-124 \mathrm{C}>\mathrm{T}$ somatic promoter mutation, when compared with family member controls (Supplemental Figure 4, A and B). We observed similar increases in the promoter mutation peak height, especially for cell lines cultured over long periods of time (Supplemental Figure 4, C and D). As LCLs often demonstrate genomic instability, we suspect that aneuploidy and cellular heterogeneity lead to enrichment of the mutant allele.

These data suggest that spontaneous acquisition of TERT promoter mutations are selected for in the somatic blood cells of individuals with short telomere lengths and heterozygous germ- 
A

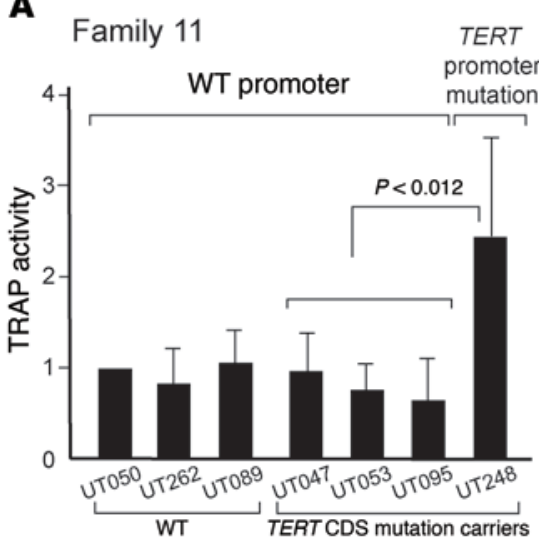

B

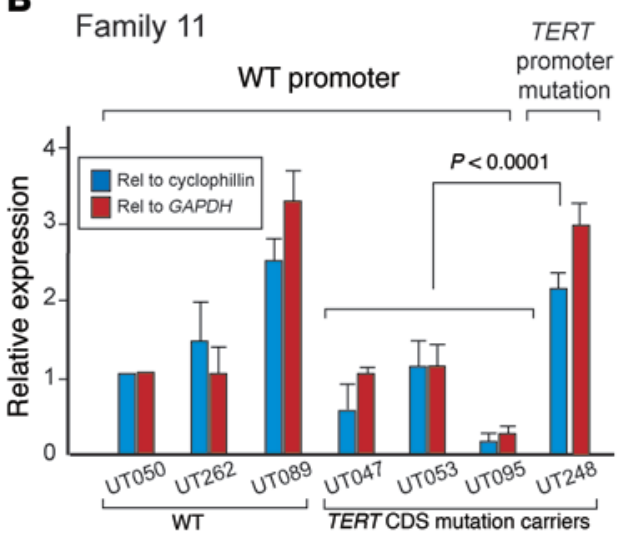

C
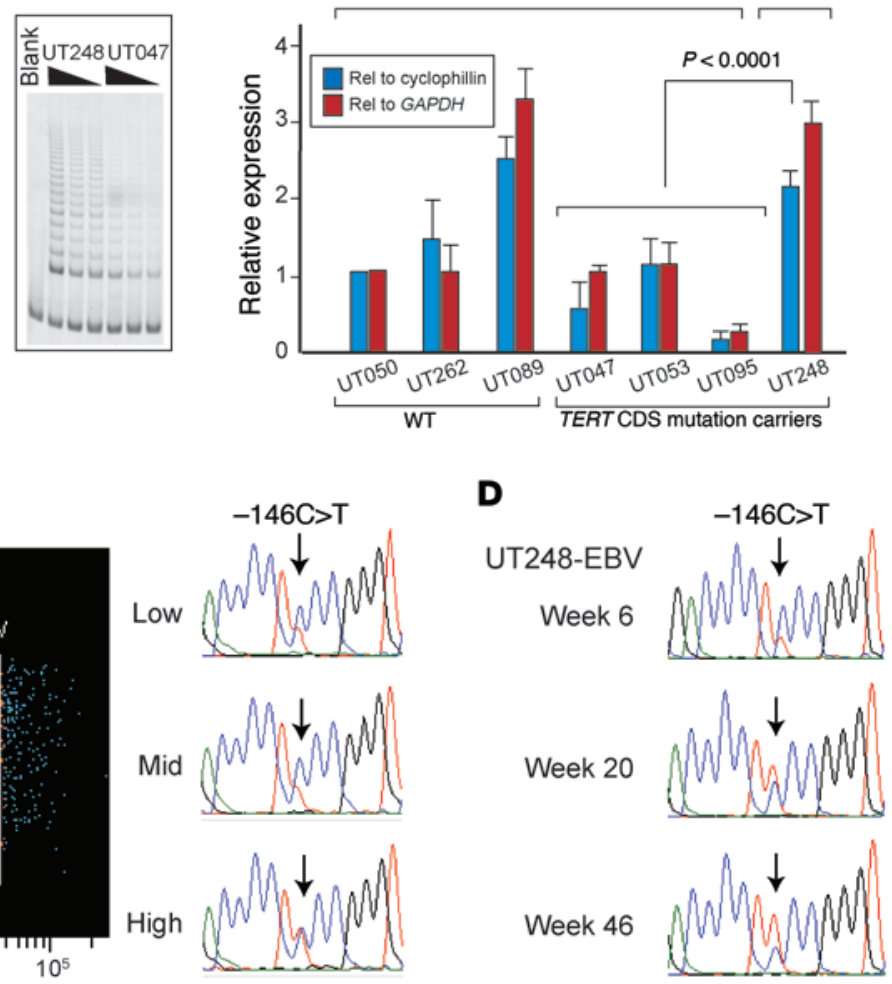

Figure 3. TERT promoter mutation is associated with increased telomerase activity and a higher proliferative capacity. (A) Telomeric repeat amplification protocol (TRAP) activity of equivalent numbers of EBV-transformed lymphoblastoid cells derived from different individuals. Data are expressed relative to the activity of a WT control and represent the mean of 5 experiments. A representative gel is shown in the inset. $P$ values were significant after correcting for multiple comparisons. (B) Expression of human TERT by real-time qPCR. Data are expressed relative to a WT control and represent the mean of triplicate experiments from 1 of 3 representative experiments. Data in A and B were analyzed by a 2-tailed Student's $t$ test. Rel, relative. (C) Flow sorting of the UT248-EBV cell line to separate cells according to their proliferative capacity, followed by sequencing of the TERT promoter. SSC, side scatter. (D) Sequencing of the TERT promoter from DNA purified from the UT248-EBV cell line at different time points. The position of the -146 variant is marked by arrows in $\mathbf{C}$ and $\mathbf{D}$.

line mutations in TERT or PARN. Since these mutations are not found in normal cohorts of similar or advanced age, there is probably positive selection for the acquisition of these somatic mutations. The 10 individuals in whom these mutations were found have a germline mutation in 1 of 2 telomere-related genes. Four of the germline coding mutations are clear loss-of-function alleles, which result from splicing, frameshift, or premature truncation mutations. Three germline TERT missense alleles, Lys570Asn, Arg631Gln, and Arg865His, are associated with a severe reduction of telomerase activity and processivity $(8,12,13)$. Another, Val144Met, is unable to elongate telomeres due to its defective association with the telomere-binding protein tripeptidyl peptidase 1 (TPP1) and ineffective recruitment to telomeres (14).

Increased telomerase activity has been shown to be a nearly ubiquitous feature in human cancers. Telomerase upregulation has been attributed to several mechanisms, including genetic amplification of the TERT locus, epigenetic deregulation, and point mutations within the promoter. The TERT promoter mutations upstream of the transcriptional start site are found in many different malignancies and are considered to be driver mutations rather than passenger events $(15,16)$. These mutations are associated with resistance to standard therapies, metastasis, recurrence, and poorer survival (17). As is seen in cancer studies, the frequency of the $-124 \mathrm{C}>\mathrm{T}$ mutation (80\%) is usually overrepresented compared with the frequency of the $-146 \mathrm{C}>\mathrm{T}$ promoter mutation (20\%). To our knowledge, these somatic TERT promoter mutations have not been previously described in human cohorts without cancer. None of the individuals with a TERT promoter mutation in this study had a personal history of cancer, but they will need to be carefully monitored for neoplastic events, as cancers are found at a higher rate in patients with DC than in the general population (18). It is possible that these somatic mutations may be better tolerated in certain cell types, such as granulocytes or $\mathrm{B}$ cells, in individuals with germline telomere-related mutations.

Hematopoietic stem/progenitor cells are dividing continuously and accumulating rare random mutations, many of which are age related and associated with clonal expansion (19). In subjects with germline mutations in the telomerase complex, somatic TERT promoter mutations are likely selected by the advantages they provide for self-renewal and proliferation. As granulocytes 
and monocytes are short-lived cells, the promoter mutations have probably been acquired by progenitors that have undergone clonal expansion. During infection or vaccination, B cells with the highest affinity for antigen undergo clonal expansion and outproliferate the lower-affinity cells. Future studies would be needed to determine whether the TERT promoter mutations are linked to clonal V (D)J recombination. This study is congruent with the previously described skewed $\mathrm{X}$-inactivation, somatic reversion, and clonal hematopoiesis found in patients with DC $(20,21)$. However, in contrast to the revertant mosaicism that leads to spontaneous correction or deletion of a pathogenic allele, here we made multiple independent observations of an activating promoter mutation on the contralateral (WT) allele. In 2 individuals (UT248 and UT822), the presence of 2 different promoter mutations linked to the WT allele underscores the selective force for these compensatory mutations to counteract progressive telomere shortening.

Although rare, these patients provide evidence of noncoding gain-of-function mutations that counterbalance germline loss-offunction mutations. The net effect of these opposing genetic forces likely leads to normalized telomerase activity. Evidence from a recent study demonstrating telomere elongation in response to danazol suggests pharmacologic activation of the telomerase promoter (22). Male sex hormones are known to upregulate telomerase and have been used to treat bone marrow failure, even for patients with DC (23). Additional investigation is warranted to determine the efficacy of telomerase activators in patients with short telomere syndromes, including those with pulmonary fibrosis. The persistence of the somatic mutation for at least 10 years in 1 patient provides genetic evidence that increased telomerase activation may be clinically safe in the setting of germline TERT coding mutations.

\section{Methods}

The Methods are detailed in the Supplemental Methods.

Statistics. All data are presented as the mean \pm SD. Two-tailed Student's $t$ tests and Fisher's exact tests were used to generate $P$ values.

Study approval. The study was approved by the Institutional Review Board of the University of Texas Southwestern Medical Center.

\section{Author contributions}

RCW, NSCvO, and CKG designed experiments; LM performed sequencing, limiting dilution, TRAP, qPCR, and proliferation experiments. YY performed the limiting dilution assay. $\mathrm{CB}$ sequenced the centenarian participants. CAN measured telomere lengths. AY performed sequencing and allelic discrimination. CKG wrote the manuscript. All authors interpreted the data and contributed to the final version of the manuscript.

\section{Acknowledgments}

The authors wish to thank the patients and their families for their participation; H.H. Hobbs for the Dallas Heart Study 2 samples; and Angie Mobley and Nicholas Loof at UT Southwestern Medical Center for flow cytometry assistance. This work was supported by grants from the Burroughs Welcome Foundation (to RCW) and the NIH (T32HL098040, to CAN; UL1TR001105, to CAN and CKG; K08CA164047, to RCW; and R01HL093096, CKG).

Address correspondence to: Christine Kim Garcia or Richard C. Wang, MSC 8591 (C.K. Garcia); MSC 9069 (R.C. Wang), 5323 Harry Hines Boulevard, Dallas, Texas 75390, USA. Phone: 214.648.1600; E-mail: Christine.garcia@utsouthwestern.edu (C.K. Garcia). Phone: 214.648.3430; E-mail: Richard.wang@ utsouthwestern.edu.
1. Greider CW, Blackburn EH. Identification of a specific telomere terminal transferase activity in Tetrahymena extracts. Cell. 1985;43 (2 Pt 1):405-413.

2. Shay JW. Role of Telomeres and Telomerase in Aging and Cancer. Cancer Discov. 2016;6(6):584-593.

3. Vinagre J, et al. Frequency of TERT promoter mutations in human cancers. Nat Commun. 2013;4:2185.

4. Bell RJ, et al. Cancer. The transcription factor GABP selectively binds and activates the mutant TERT promoter in cancer. Science. 2015;348(6238):1036-1039.

5 . Borah S, et al. Cancer. TERT promoter mutations and telomerase reactivation in urothelial cancer. Science. 2015;347(6225):1006-1010.

6. Chiba K, Johnson JZ, Vogan JM, Wagner T, Boyle JM, Hockemeyer D. Cancer-associated TERT promoter mutations abrogate telomerase silencing. Elife. 2015;4:e07918.

7. Armanios MY, et al. Telomerase mutations in families with idiopathic pulmonary fibrosis. N Engl JMed. $2007 ; 356$ (13):1317-1326.

8. Tsakiri KD, et al. Adult-onset pulmonary fibrosis caused by mutations in telomerase. Proc Natl Acad Sci USA. 2007;104 (18):7552-7557.

9. Stuart BD, et al. Exome sequencing links muta- tions in PARN and RTEL1 with familial pulmonary fibrosis and telomere shortening. Nat Genet. 2015;47 (5):512-517.

10. Stanley SE, et al. Loss-of-function mutations in the RNA biogenesis factor NAF1 predispose to pulmonary fibrosis-emphysema. Sci Transl Med. 2016;8 (351):351ra107.

11. Newton CA, et al. Telomere-related lung fibrosis is diagnostically heterogeneous but uniformly progressive. Eur Respir J. 2016;48 (6):1710-1720

12. Diaz de Leon A, et al. Telomere lengths, pulmonary fibrosis and telomerase (TERT) mutations. PLoS One. 2010;5 (5):e10680.

13. Xin ZT, et al. Functional characterization of natural telomerase mutations found in patients with hematologic disorders. Blood. 2007;109 (2):524-532.

14. Zhong FL, Batista LF, Freund A, Pech MF, Venteicher AS, Artandi SE. TPP1 OB-fold domain controls telomere maintenance by recruiting telomerase to chromosome ends. Cell. 2012;150 (3):481-494.

15. Horn S, et al. TERT promoter mutations in familial and sporadic melanoma. Science. 2013;339 (6122):959-961.

16. Huang FW, Hodis E, Xu MJ, Kryukov GV, Chin
L, Garraway LA. Highly recurrent TERT promoter mutations in human melanoma. Science. 2013;339 (6122):957-959.

17. Liu T, Yuan X, Xu D. Cancer-Specific Telomerase Reverse Transcriptase (TERT) Promoter Mutations: Biological and Clinical Implications. Genes (Basel). 2016;7(7):E38.

18. Alter BP, Giri N, Savage SA, Rosenberg PS. Cancer in dyskeratosis congenita. Blood. 2009;113(26):6549-6557.

19. Xie M, et al. Age-related mutations associated with clonal hematopoietic expansion and malignancies. Nat Med. 2014;20 (12):1472-1478.

20. Vulliamy TJ, Knight SW, Dokal I, Mason PJ. Skewed $\mathrm{X}$-inactivation in carriers of $\mathrm{X}$-linked dyskeratosis congenita. Blood.1997;90 (6):2213-2216.

21. Jongmans MC, et al. Revertant somatic mosaicism by mitotic recombination in dyskeratosis congenita. Am J Hum Genet. 2012;90 (3):426-433.

22. Townsley DM, et al. Danazol Treatment for Telomere Diseases. N Engl J Med. 2016;374(20):1922-1931.

23. Khincha PP, Wentzensen IM, Giri N, Alter BP, Savage SA. Response to androgen therapy in patients with dyskeratosis congenita. Br J Haematol. 2014;165 (3):349-357. 